\title{
El contexto del nacimiento en los años cuarenta en una zona rural del sur de España
}

\section{Birth context in the forties in a rural area of southern Spain}

Contexto do nascimento em quarenta anos em uma

\section{área rural do sul da Espanha}

María de los Ángeles Castaño Molina ${ }^{1}$, María Emilia Martínez Roche²,

Natalia Emilia Rodríguez Portilla ${ }^{3}$ y César Carrillo García ${ }^{4}$

${ }^{1}$ Dra. en Antropología. Universidad de Murcia

${ }^{2}$ Dra. en Pedagogía. Universidad de Murcia.

${ }^{3}$ Dra. en Enfermería. Universidad de Murcia.

${ }^{4}$ Dr. en Enfermería. Universidad de Murcia.

Cómo citar este artículo en edición digital: Castaño Molina, M.A., Martínez Roche, M.E., Rodríguez Portilla, N.E., \& Carrillo García, C. (2018). El contexto del nacimiento en los años cuarenta en una zona rural del sur de España. Cultura de los Cuidados (Edición digital), 22(50). Recuperado de http://dx.doi.org/10.14198/cuid.2018.50.10

Correspondencia: Facultad de Enfermería. Campus Universitario de Espinardo. CP 30100 Murcia.

Correo electrónico: angcastano@hotmail.com

Recibido: 12/06/2017; Aceptado: 03/11/2017

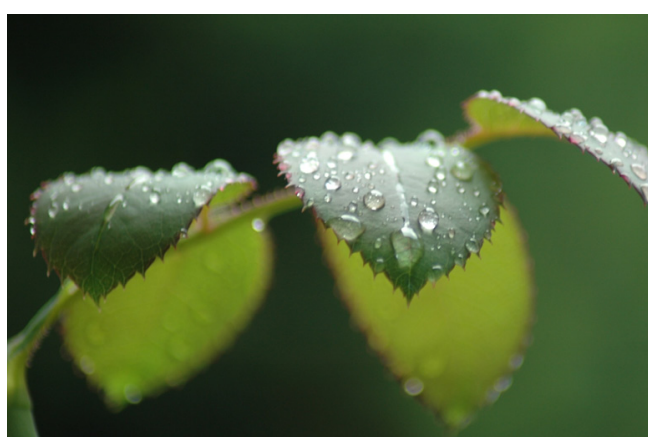

\section{ABSTRACT}

In this study we set objectives to define the birth context of a rural town in Spain in the post-war period 1945-1950; to define what influence gender roles had on experience of these events; to identify what values and beliefs had influence on the childbirth process and to investigate which attitudes promoted change. Taking a qualitative approach, we chose an ethnographic method. The information was obtained through life story reports and semistructured interviews during 2009. The study was carried out in a rural town in southern Spain: Abarán, in the Region of Murcia. Elev- en life stories were collected from women who became mothers between 1945 and 1950 and five semi-structured interviews were also carried out to enable triangulation and information expansion.After transcription of the interviews, the information was fragmented and, after thematic analysis of the content, three categories were defined: household work/ economy, marriage/family and childbirth. Despite the way that gender roles defined the lives of men and women. Even though the social model of the time links women to activities at home, these women also worked hard at agricultural labour outside the home. The prevailing beliefs meant that many of the women were ignorant of their own bodies, even up until the moment of giving birth. Midwives were the reference professionals for this event.

Key words: Midwife, childbirth, work, family, gender

\section{RESUMEN}

En este estudio nos planteamos como objetivos conocer el contexto del nacimiento en 
un pueblo rural de España en un período de posguerra, 1945-1950; determinar cómo influyeron los roles de género en la vivencia de estos acontecimientos; identificar qué valores $y$ creencias influyeron en el proceso del nacimiento de sus hijos y descubrir qué actitudes promovieron cambios.

Desde el enfoque cualitativo hemos escogido el método etnográfico. La información se obtuvo a través de historias de vida y entrevistas semiestructuradas durante 2009.

El estudio se llevó a cabo en un pueblo rural del sureste de España (Abarán-Murcia). Se realizaron 11 historias de vida a mujeres que fueron madres entre 1945 y 1950 y 5 entrevistas semiestructuradas para la triangulación y ampliación de la información. Tras la trascripción y fragmentación de las entrevistas y mediante análisis temático del contenido surgieron tres categorías: trabajo/economía doméstica, matrimonio/familia y nacimiento. Los roles de género se han impuesto en la vida de los hombres y las mujeres. El modelo social relaciona a la mujer con las actividades dentro del hogar, sin embargo, la mujer trabajaba duro fuera de casa en labores agrícolas. Las creencias transmitidas provocaban que las mujeres no conocieran su propio cuerpo incluso en el momento del parto, la matrona es el personal de referencia para este acontecimiento.

Palabras clave: Matrona, nacimiento, trabajo, familia, género.

\section{RESUMO}

Este estudo tem como objectivos conhecer o contexto do nascimento numa vila rural de Espanha no período de pós-guerra, 19451950; determinar como o papel de género influiu na vivência desses eventos; identificar quais valores e crenças influíram no processo do nascimento dos filhos e descobrir quais actitudes promoveram as mudanças. A partir da abordagem qualitativa foi escolhido o método etnográfico. As informações foram obtidas através das histórias de vida e entrevistas semi-estruturadas ao longo de 2009. O estudo desenvolveu-se numa vila rural no sudeste da Espanha (Abarán, Murcia). Foram realizadas onze histórias de vida a mulheres que foram mães entre 1945 e 1950, e cinco entrevistas semi-estruturadas para a triangulação e ampliação da informação. Após a transcrição e fragmentação das entrevistas e da análise temática do conteúdo, surgiram três categorias: trabalho/economia doméstica, casamento/ família e nascimento. $\mathrm{O}$ papel do género tem-se imposto na vida dos homens e das mulheres. $\mathrm{O}$ modelo social relaciona à mulher com as actividades dentro de casa, no entanto, a mulher trabalhava duramente fora de casa na agricultura. As crenças transmitidas fizeram com que as mulheres não conhecessem o seu próprio corpo, mesmo no momento do parto, sendo a parteira a pessoa de referência para este acontecimento.

Palavras chave: Parteira, nascimento, trabalho, família, género.

\section{INTRODUCCIÓN}

En 1945 nos encontramos con las consecuencias de la Guerra Civil española (19361939) y la Segunda Guerra Mundial en Europa. Las pérdidas económicas y humanas provocan pobreza en la mayor parte de la población. En España se vive una dictadura política con autarquía económica y racionamiento de alimentos, además la legislación propone un modelo de mujer abnegada al marido centrada en las tareas domésticas y madre de muchos hijos (Sarasúa y Molinero, 2009; Gómez-Ferrer, 2011).

El estudio se planteó con mujeres de Aba- 
rán (Murcia-España), es un pueblo rural de clima mediterráneo y con un río llamado Segura que hace posible el cultivo de frutales. En los años cuarenta y, todavía hoy, los frutales son la base de su economía. Las empresas de exportación y conservas de frutas (sobre todo melocotón, albaricoque, uva y ciruela) daban trabajo a todo el pueblo, en el campo trabajaban en su mayoría hombres y en las fábricas el $80 \%$ de los trabajadores eran mujeres. El acceso a los servicios sanitarios era caro y dificultoso $\mathrm{y}$, aunque había quien se podía pagar un dinero anual para asistencia médica, la beneficencia (ayuda social) debía ocuparse de la asistencia sanitaria en gran parte de la población (Castaño, 2013). Durante el parto y el puerperio la matrona era el profesional de referencia para las mujeres y su familia, este proceso vital se vivían en los domicilios (Sandall et al., 2009; Fenwick et al., 2010; Steenkamp et al., 2011; Hoang y Le, 2012; Likis, 2012; Redondo et al., 2012).

En este contexto, la naturalización de los roles ha provocado desigualdad de oportunidades entre los hombres y las mujeres a lo largo de su ciclo vital, la forma en que se vive y organiza la reproducción nos informa del tipo de sociedad en la que nos encontramos, porque este acontecimiento lleva implícito las formas de relación del individuo tanto a nivel comunitario como en su entorno familiar (Collado, 2009; Carosio, 2010; Carrasco et al., 2011; Martínez, 2012). Las instituciones estatales son las que proponen y propagan el modelo de sociedad deseado y, por lo tanto, lo que hombres y mujeres deben realizar en ella. El hecho de poder explicar la construcción de la realidad de las mujeres en su parto, hace necesario conocer todos los aspectos de su vida (Esteban, 2006; Klinge, 2010) y, derivados de las categorías que subyacen de las entrevistas, hemos analizado el trabajo, la formación académica/profesional y la familia.

\section{METODOLOGÍA}

Es un estudio cualitativo para conocer la vivencia del nacimiento entre 1945 a 1950 ya que en 1944 se aprueba una nueva Ley de Sanidad. En 2009 se realizaron 11 historias de vida y 5 entrevistas semiestructuradas para la triangulación de los datos y ampliación de la información. Las preguntas guía de las entrevistas se agruparon en 4 grupos: experiencias de la niñez, costumbres de la adolescencia, vivencias de su juventud y visión actual de los acontecimientos de la vida.

Tras la trascripción y fragmentación de las entrevistas y mediante análisis temático se examinaron los datos por tres investigadores de forma independiente y sin programa informático, de ahí surgieron tres categorías:

Categoría 1: Organización del trabajo/ economía doméstica. Esta categoría surge de agrupar las subcategorías: Trabajo de la mujer fuera de casa; trabajo del marido fuera de casa; trabajo en casa de la mujer; trabajo en casa del marido.

Categoría 2: Matrimonio/Familia. Influencia del modelo social establecido de familia y mujer que va a determinar el resto de relaciones sociales y la reproducción.

Categoría 3: Nacimiento, que engloba las subcategorías: conocimiento del cuerpo y proceso del nacimiento (embarazo, parto y puerperio).

\section{RESULTADOS}

Categoría 1: Organización del trabajo/Economía doméstica.

El modelo social relaciona al hombre con el trabajo fuera de casa y a la mujer con las actividades dentro del hogar, sin embargo, las mujeres trabajaban duro fuera de casa en labores 
agrícolas, aún así, su trabajo y salario son considerados sólo como complemento al del marido.

Realizábamos tareas de hombres y mujeres, cuando llegábamos de trabajar fregábamos, traíamos agua a casa y así hasta la noche. Ellos iban por leña. No teníamos horario, era una vida muy dura (participante 1).

La separación de tareas productivas y reproductivas también las podemos ver reflejadas en los siguientes testimonios durante el parto:

Los hombres se quedaban fuera de la habitación esperando a que nacieran los hijos. Ellos avisaban a la comadrona, encendían el fuego... (participante 5).

Después del parto él se va a trabajar como siempre y nosotras en casa con nuestras tareas, igual que siempre (participante 1).

El trabajo agrícola de las mujeres era eventual, variaba según la producción de la cosecha. Las necesidades económicas obligaban a la mujer a trabajar fuera de casa, por ello, tras el parto, se incorporaban al trabajo lo antes posible.

\section{Categoría 2: Familia}

Los acontecimientos más destacados de la vida de estas mujeres han sido casarse y tener hijos; para conservar el honor de la familia se comportan como dictan las normas sociales e identifican el matrimonio necesario para ser madres.

Su formación se centraba en las labores domésticas, la formación académica recibida ha sido escasa, algunas no tuvieron la oportunidad de poder aprender a leer y escribir, sin embargo, las que pudieron acceder a los estudios los abandonaron para casarse, otras para trabajar y ayudar económicamente a su familia.

Madre, te quiero mucho pero no te perdono que no me dejaras estudiar. Era mi mayor ilusión y me obligaste a trabajar (participante 6).
Me gustaba mucho leer, por las noches tapaba con una toalla las rendijas de la puerta de mi habitación para que mis padres no vieran la luz y me obligaran a apagarla (participante 9).

\section{Categoría 3: Nacimiento}

Los valores y creencias de la época provocaron que las mujeres no conocieran su cuerpo, siendo la salud sexual y reproductiva tema tabú.

A las niñas no nos decían nada, entre nosotras a veces comentábamos algo u oíamos algo de los mayores (participante 5).

Las madres nos decían: cuando seas mayor te enterarás, cuando llegue el momento lo sabrás (participante 1).

En aquella época las chicas no comentábamos con los chicos nada. Eso era tabú (participante 4).

El nacimiento de los hijos se vivía en los domicilios y se divide en tres fases: en la primera las mujeres se apartan a un espacio íntimo y privado dentro de su hogar, suele ser la habitación matrimonial, pero antes, como "mujeres modelo" han limpiado toda la casa. El marido suele quedar fuera de la habitación y es el encargado de encender el fuego, llamar a la comadrona y atender a las personas que les acompañan.

Para el parto (segunda fase) en las casas se solía preparar paños limpios y agua caliente, la matrona llevaba el resto del material (pinzas para el cordón, las tijeras...). El nacimiento tenía lugar en la cama, en un colchón en el suelo e incluso entre dos sillas, adoptando la posición más cómoda para la mujer.

Tras el parto (tercera fase) se solía tirar la placenta a la acequia y el tiempo de descanso en casa variaba según las necesidades económicas del hogar. La comida escaseaba, pero la familia llevaba a la madre guiso de gallina, el 
cual servía para su recuperación y estimulación para la producción de leche.

Las creencias religiosas hacían que las mujeres se encomendaran a los Santos y, la primera vez que salían de casa tras el nacimiento de su hijo, se dirigían a la iglesia para agradecer a Dios que todo hubiera ido bien.

Los recién nacidos de esta época (ahora abuelos) precisaban de menos recursos materiales que los de hoy; el ajuar era cosido por sus madres o encargado a las modistas del pueblo, algunos no tenían cuna, pero la mayoría eran dormidos en mecedoras de madera. La alimentación se basaba en la lactancia materna y, en el caso de no poderla ofrecer se daba papilla de harina y el caldo de cocer arroz; en ocasiones, las mujeres más adineradas contrataban a un ama de cría. Debemos destacar a las llamadas madres de leche, son mujeres que amamantan a hijos de otras madres sin pedir nada a cambio, en esta época de escasez su dedicación es modelo de solidaridad.

El profesional de referencia para el proceso de nacimiento era la matrona, la cual atendía a la madre y a su hijo en el domicilio hasta unos días después del parto. Sólo cuando se complicaba el proceso la mujer era trasladada al hospital de la ciudad para realizarle una cesárea.

Cuando nos poníamos con dolores se avisaba a la comadrona y no hacía falta nada más. (participante 5).

Mis cinco hijos han nacido en mi casa tranquilamente con la comadrona (participante 6).

\section{DISCUSIÓN}

Las mujeres siguen el modelo social patriarcal establecido por el sistema político y el catolicismo, no se pueden divorciar y si desean trabajar fuera de casa necesitan la autorización de su marido. La formación tradicional para las mujeres era el trabajo doméstico y muy

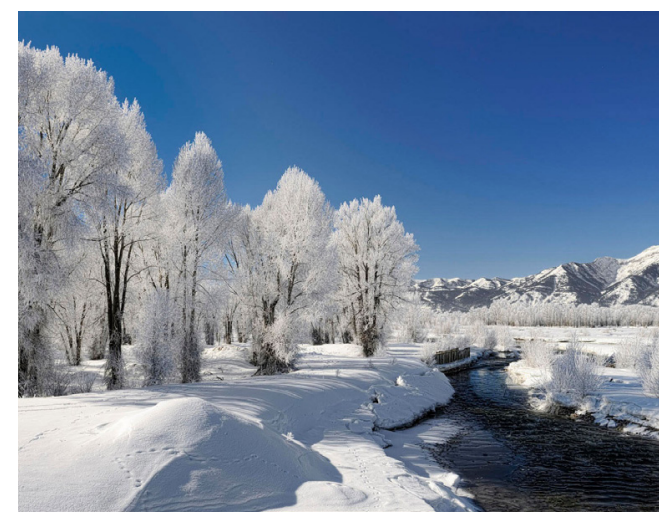

pocas accedían a estudios superiores además, desde 1938, la educación de los niños y las niñas se realiza por separado (Sarasúa y Molinero, 2009; Gómez-Ferrer, 2011). El trabajo se desarrolla en contextos determinados donde se expresan las relaciones sociales existentes (Comas, 1995; Téllez y Martínez, 1997) por lo tanto, el trabajo puede ser visible o invisible, como por ejemplo el doméstico. Las mujeres de nuestro estudio no asumen en su totalidad el modelo social establecido, sino que por necesidades económicas deben trabajar fuera de casa al mismo tiempo que cuidan de toda la familia y realizan las tareas domésticas. Por ambos motivos su trabajo se invisibiliza y se infravalora incluso cuando es asalariado, considerándose un complemento al salario del marido (Collado, 2009; Carosio, 2010; Carrasco et al., 2011; Martínez, 2012).

Concluimos que las mujeres cambian el modelo social por necesidad y su reacción es la que provoca el cambio social. La transformación de modelos hegemónicos que conforman las estructuras y el surgimiento de otros como consecuencia de nuevas experiencias de interacción social, da lugar a nuevas socializaciones (nuevos comportamientos) que apuntan a otras miradas socio-culturales y con ellas cambios en el sistema de género. Los nuevos modelos tendrán más fuerza cuanto más in- 
tención haya de provocar un cambio (Del Valle et al., 2002; Cerri, 2010).

La evidencia confirma que las mujeres precisan la asistencia ofrecida por las matronas en el parto y el puerperio, siendo de gran importancia para cubrir sus necesidades sociosanitarias (Sandall et al., 2009; Fenwick et al., 2010; Steenkamp et al., 2011; Hoang y Le, 2012; Likis, 2012; Redondo et al., 2012). Durante los años de estudio se comprueba que la mortalidad materno infantil en el pueblo es inferior a la media nacional (Castaño, 2013) y en esto influye la asistencia de las matronas en el domicilio.

\section{CONCLUSIONES}

Para el estudio de cualquier aspecto de la vida de las personas hay que considerar la relación con su entorno y sus experiencias vividas. Los roles de género han determinado el contexto en el que han vivido los hombres y mujeres reflejándose en el trabajo, la familia y el cuerpo, sin embargo, las mujeres del pueblo han sido motores de cambio para las siguientes generaciones. Aunque muchas de ellas no pudieron vivir de otra forma, reconocen la importancia de la formación, su trabajo y esfuerzo tanto dentro como fuera del hogar, así como el desconocimiento de su propio cuerpo. Este contexto ha determinado tanto la forma de producción de los miembros de las familias, como la forma de reproducción siguiendo un modelo patriarcal en el que la mujer es cuidadora de los hijos y el marido dentro del hogar. El nacimiento es un acontecimiento social que tiene lugar en el seno de la familia y en su domicilio en el que la comadrona ayuda hasta que madre e hijo se han recuperado.

\section{Agradecimientos}

A todas las mujeres que han hecho posible este trabajo y a sus familias.

\section{BIBLIOGRAFÍA}

- Carosio, A. (2010). El trabajo de las mujeres: desigualdad, invisibilidad y explotación. Revista Venezolana de Estudios de la Mujer, 15, 7-13.

- Carrasco, C., Borderías, C., y Torns T. (2011) El trabajo de cuidados. Historia, teoría y políticas. Madrid: Los libros de la Catarata.

- Castaño Molina, M.A. (2013) El parto como construcción cultural de las mujeres abaraneras, 1945-1950. Málaga: Ediciones Atenea.

- Cerri, C. (2010). La subjetividad de género. El sujeto sexuado entre individualidad y colectividad. Gazeta de Antropología, 26,10-16.

- Collado, P.A. (2009). Visibilidad e invisibilidad: Acerca del trabajo y las mujeres. Revista Katálysis, 12,178-187.

- Comas D’Argemir, D. (1995). Trabajo, género, cultura. La construcción de desigualdades entre hombres y mujeres. Barcelona: Icaria editorial, S.A.

- Del Valle, T., Apaolaza, J.M., Arbe, F., Cucó, J., Díez, C., Esteban, M.L., Etxebarría, F., y Maquieira, V. (2002). Modelos emergentes en los sistemas y las relaciones de género. Madrid: Narcea, S.A.

- Esteban Galarza, M.L. (2006). El Estudio de la Salud y el Género: Las Ventajas de un Enfoque Antropológico y Feminista. Salud Colectiva, 2, 9-20.

- Fenwick, J., Butt, J., Dhaliwal, S., Hauck, Y. y Schmied, V. (2010) We Australian women's perceptions of the style and quality of midwifery postnatal care in hospital and at home. Women Birth, 23, 10-21.

- Gómez- Ferrer, G. (2011) Historia de las mujeres en España: siglo XIX y XX. Madrid: Arco Libros, S.L.

- Hoang, H. y Le, Q. (2012). Trade-off bettween local access and safety considerations in chilbirth: Rural Tasmanian women's perspectives. Aust. J. Rural Health, 20,144-149.

- Klinge, I. (2010). Sex and gender in biomedicine: Promises for women and men. How incorporation of sex and gender in research will lead to a better health care. En Klinge, I. y Wiesemann C. (Eds.), Gender in biomedicine (theories, methodologies, results) (15-32). Götingen: Universidad de Götingen.

- Likis, F.E. (2012). Promoting midwife-provided gyneco- 
logic care: with women for a lifetime. J Midwifery Womens Health, 57, 545-6.

- Martínez, M. (2012). Explorando la invisibilidad de mujeres en diferentes culturas en la sociedad y en los medios de comunicación. Palabra Clave 13, 161-173.

- Redondo, S.L., Sanz Díaz, M.C., Pérez Martínez, E., Santa Cruz Martín, B., Vicente López, A. y Pacheco Ardila, F. (2012). Puesta en marcha del informe de alta emitido por matronas en el puerperio de bajo riesgo. Matronas Prof., 13, 90-96.

- Sandall, J., Hatem, M., Devane, D., Soltani, H. y Gates, S. (2009) Discussions of findings from a Cochrane review of midwife-led versus other models of care for childbea- ring women: continuity, normality and safety. Midwifery, 25, 8-13.

- Sarasúa, C., y Molinero, C. (2009). Trabajo y niveles de vida en el franquismo. Un estado de la cuestión desde una perspectiva de género. Barcelona: Icaria editorial, S.A.

- Steenkamp, M., Rumbold, A.R., Kildea, S., Bar-Zeev, S.J, Kruske, S., Dumbar, T. y Barclay, L. (2011). Measuring what matters in delivering services to remote-dwelling Indigenous mothers and infants in the Northern Territory, Australia. Aust. J. Rural Health, 20, 228-237.

- Téllez, A., y Martínez, J. (1997). Economía informal y perspectiva de género en contextos de trabajo. Barcelona: Icaria.

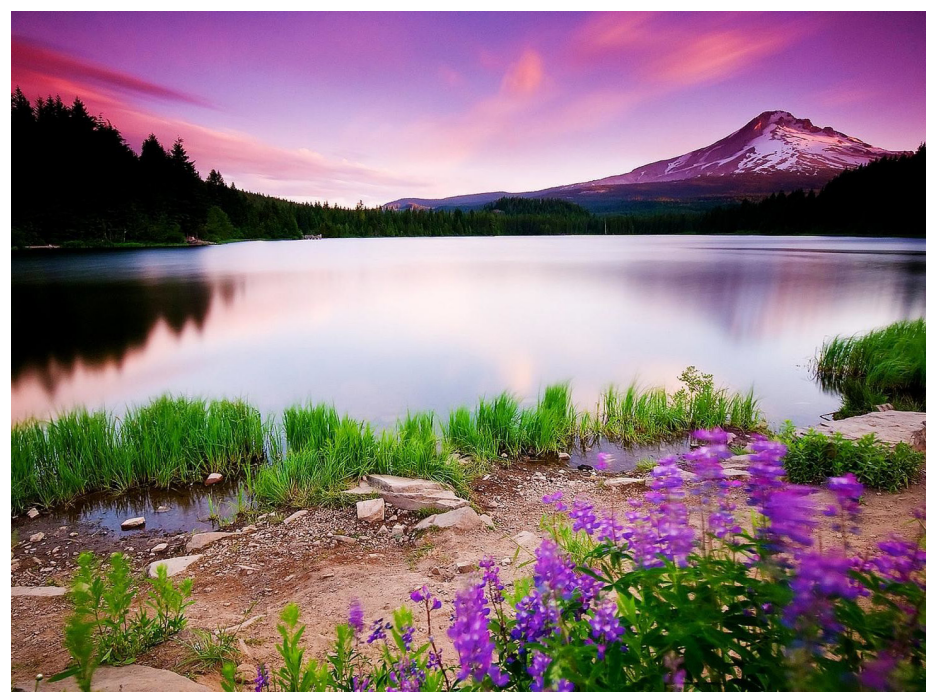

